DOI 10.4171/JEMS/233

Joachim Stubbe

\title{
Universal monotonicity of eigenvalue moments and sharp Lieb-Thirring inequalities
}

Received January 22, 2009 and in revised form February 13, 2009

Abstract. We show that phase space bounds on the eigenvalues of Schrödinger operators can be derived from universal bounds recently obtained by E. M. Harrell and the author via a monotonicity property with respect to coupling constants. In particular, we provide a new proof of sharp LiebThirring inequalities.

Keywords. Universal bounds for eigenvalues, spectral gap, phase space bounds, Lieb-Thirring inequalities, Schrödinger operators

\section{Introduction}

We consider the eigenvalues $E_{j}(\alpha)$ of a one-parameter family of Schrödinger operators

$$
H(\alpha)=-\alpha \Delta+V(x)
$$

on $\mathbb{R}^{d}$ for constants $\alpha>0$. For simplicity we suppose that $V(x)$ is a continuous function of compact support and we denote its negative part by $V_{-}(x)$. It is a well-known fact (see e.g. [3, 9] and references therein) that for all $\sigma \geq 0$,

$$
\lim _{\alpha \rightarrow 0+} \alpha^{d / 2} \sum_{E_{j}(\alpha)<0}\left(-E_{j}(\alpha)\right)^{\sigma}=L_{\sigma, d}^{\mathrm{cl}} \int_{\mathbb{R}^{d}} V_{-}(x)^{\sigma+d / 2} d x
$$

with $L_{\sigma, d}^{\mathrm{cl}}$, called the classical constant, given by

$$
L_{\sigma, d}^{\mathrm{cl}}=(4 \pi)^{-d / 2} \frac{\Gamma(\sigma+1)}{\Gamma(\sigma+d / 2+1)} .
$$

Lieb-Thirring inequalities are inequalities of the form

$$
\alpha^{d / 2} \sum_{E_{j}(\alpha)<0}\left(-E_{j}(\alpha)\right)^{\sigma} \leq L_{\sigma, d} \int_{\mathbb{R}^{d}} V_{-}(x)^{\sigma+d / 2} d x
$$

J. Stubbe: EPFL, IMB-FSB, Station 8, CH-1015 Lausanne, Switzerland; e-mail: Joachim.Stubbe@epfl.ch

Mathematics Subject Classification (2010): 81Q10, 35P15, 35P20 
for some constant $L_{\sigma, d} \geq L_{\sigma, d}^{\mathrm{cl}}$ and are widely discussed in the literature (see e.g. [3, 9, 11). A longstanding question is when (1.4) holds with $L_{\sigma, d}=L_{\sigma, d}^{\mathrm{cl}}$. The most general result is due to Laptev and Weidl [10] who proved that $L_{\sigma, d}=L_{\sigma, d}^{\mathrm{cl}}$ for all $\sigma \geq 3 / 2$ and $d \geq 1$. Their proof is based on a dimensional reduction of Schrödinger operators with operator valued potentials, which allows them to make use of the bound for $\sigma=3 / 2$, $d=1$ which has been first proven by Lieb and Thirring [12]. For a simplified proof see also [2]. On the other hand, by analyzing the spectra of harmonic oscillators Helffer and Robert [8] have shown that $L_{\sigma, d}>L_{\sigma, d}^{\mathrm{cl}}$ for $\sigma<1$ while de la Bretèche showed that these spectra are in agreement with the conjecture $L_{\sigma, d}=L_{\sigma, d}^{\mathrm{cl}}$ for $\sigma \geq 1$ [4].

Recently, Harrell and the author have established universal trace inequalities for abstract self-adjoint operators $H$ modelled on Schrödinger operators [7]. If $G$ is another self-adjoint operator, then under suitable domain conditions (see Corollary 2.3 of [7])

$$
\begin{array}{r}
\sum_{E_{j} \in J}\left(\left(z-E_{j}\right)^{2}\left\langle[G,[H, G]] \phi_{j}, \phi_{j}\right\rangle-2\left(z-E_{j}\right)\left\langle[H, G] \phi_{j},[H, G] \phi_{j}\right\rangle\right) \\
=2 \sum_{E_{j} \in J} \int_{\kappa \in J^{c}}\left(z-E_{j}\right)(z-\kappa)\left(\kappa-E_{j}\right) d G_{j \kappa}^{2}
\end{array}
$$

where $J$ denotes a subset of the discrete spectrum of $H$ and $J^{c}$ its complement and the measure $d G_{j \kappa}^{2}$ corresponds to the matrix elements of the operator $G$ with respect to the spectral projections onto $J$ and $J^{c}$ (see [7] for the details). Exploiting this identity we prove the following

Theorem 1.1. Let $V(x)$ be a continuous function of compact support. Then the mapping

$$
\alpha \mapsto \alpha^{d / 2} \sum_{E_{j}(\alpha)<0}\left(-E_{j}(\alpha)\right)^{2}
$$

is non-increasing for all $\alpha>0$. Consequently,

$$
\alpha^{d / 2} \sum_{E_{j}(\alpha)<0}\left(-E_{j}(\alpha)\right)^{2} \leq L_{2, d}^{\mathrm{cl}} \int_{\mathbb{R}^{d}} V_{-}(x)^{2+d / 2} d x
$$

for all $\alpha>0$.

The link between universal inequalities and semiclassical estimates has been first made in [6] where it has been shown for the Dirichlet Laplacian $-\Delta_{D}$ on a bounded domain $D \subset \mathbb{R}^{d}$ that the mapping

$$
t \mapsto t^{d / 2} \operatorname{tr}\left(e^{-t \Delta_{D}}\right)
$$

is always decreasing and therefore bounded by its semiclassical limit, that is,

$$
\operatorname{tr}\left(e^{-t \Delta_{D}}\right) \leq(4 \pi t)^{-d / 2}|D| .
$$

Harrell and Hermi have extended this technique to Riesz means of the Dirichlet Laplacian [5]. In [7] it has been pointed out that the monotonicity of mappings like (1.8] is a 
universal property of a large family of "trace-controllable" functions (as precisely defined in [7]) of Schrödinger operators and we shall derive in the present paper a corresponding universal property of one-parameter families of Schrödinger operators. Our second result extends this property to Schrödinger operators of the form 1.1) with confining potentials $V(x)$ such that

$$
\int_{\mathbb{R}^{d}} e^{-t V(x)} d x<\infty
$$

for all $t>0$. We provide a monotonicity result implying the Golden-Thompson inequality (see e.g. [13]) for Schrödinger operators (1.1]:

Theorem 1.2. If 1.10 holds, then for all $t>0$ the mapping

$$
\alpha \mapsto \alpha^{d / 2} \operatorname{tr}\left(e^{-t H(\alpha)}\right)
$$

is non-increasing for all $\alpha>0$. Consequently, for all $\alpha>0$,

$$
\operatorname{tr}\left(e^{-t H(\alpha)}\right) \leq(4 \pi \alpha t)^{-d / 2} \int_{\mathbb{R}^{d}} e^{-t V(x)} d x<\infty .
$$

\section{Proof of main results}

The key for proving our main results is the trace formula for self-adjoint operators proved in [7]. For convenience we reformulate this result for the operator $H(\alpha)$ in a slightly different and, as we believe, more transparent way. To make the present paper self-contained we give an elementary proof of the trace formula. For simplicity, we consider only the case of purely discrete spectra (more relevant for Theorem 1.2). In the presence of continuous spectrum one uses the spectral integral as in [7].

Theorem 2.1 (Trace formula for $H(\alpha)$ ). Suppose that $H(\alpha)$ given in (1.1) has a spectrum consisting of eigenvalues $E_{k}=E_{k}(\alpha)$ with associated eigenfunctions $\phi_{k}$ forming an orthonormal basis of the underlying Hilbert space $L^{2}\left(\mathbb{R}^{d}\right)$. Then for any function $f: \mathbb{R} \rightarrow \mathbb{R}$,

$$
d \sum_{E_{j}} f\left(E_{j}\right)+2 \alpha \sum_{E_{j} \neq E_{k}} T_{j k} \frac{f\left(E_{k}\right)-f\left(E_{j}\right)}{E_{k}-E_{j}}=0
$$

provided all sums are finite where

$$
T_{j k}=T_{k j}=\left|\int_{\mathbb{R}^{d}} \phi_{j} \nabla \phi_{k} d x\right|^{2}
$$

denote the kinetic energy matrix elements. 
Proof. Let $x_{a}, a=1, \ldots, d$, denote cartesian coordinates in $\mathbb{R}^{d}$ and $D_{a}=\partial / \partial x_{a}$. The first identity we derive in the following is due to canonical commutation (or integration by parts) and the completeness of eigenfunctions. Indeed, for all $j$,

$$
1=-2 \int_{\mathbb{R}^{d}} x_{a} \phi_{j} D_{a} \phi_{j} d x=-2 \sum_{k} \int_{\mathbb{R}^{d}} x_{a} \phi_{j} \phi_{k} d x \int_{\mathbb{R}^{d}} \phi_{k} D_{a} \phi_{j} d x .
$$

Taking the scalar product of $H(\alpha) \phi_{j}=E_{j} \phi_{j}$ with $\phi_{k}$ and vice versa we derive, after subtracting both expressions, the gap formula

$$
\left(E_{k}-E_{j}\right) \int_{\mathbb{R}^{d}} x_{a} \phi_{j} \phi_{k} d x=-2 \alpha \int_{\mathbb{R}^{d}}\left(D_{a} \phi_{j}\right) \phi_{k} d x .
$$

We note that the r.h.s. is zero for degenerate eigenvalues. Therefore after summing over all coordinates in 2.3 we get the sum rule

$$
d=4 \alpha \sum_{E_{k} \neq E_{j}} \frac{T_{j k}}{E_{k}-E_{j}} .
$$

Multiplying 2.5 by $f\left(E_{j}\right)$, summing over $j$ and symmetrizing the double sum we finally obtain 2.1.

Applying Theorem 2.1 to $f(E)=(z-E)^{2}$ for $E<z$ and $f(E)=0$ otherwise we recover (1.5) with $G$ being the multiplication operator $x_{a}$ after summing over all coordinates as shown in [6, 7]:

$$
\sum_{E_{j}<z}\left(d\left(z-E_{j}\right)^{2}-4 \alpha\left(z-E_{j}\right) T_{j}\right)=4 \alpha \sum_{E_{j}<z} \sum_{E_{k} \geq z} T_{j k} \frac{\left(z-E_{j}\right)\left(z-E_{k}\right)}{E_{k}-E_{j}}
$$

with

$$
T_{j}=\sum_{E_{k}} T_{j k}=\int_{\mathbb{R}^{d}}\left|\nabla \phi_{j}\right|^{2} d x
$$

Remark 2.2. Formula 2.5 can also be easily derived from second order perturbation theory. Indeed, for a fixed vector $v \in \mathbb{R}^{d}$ consider the operator $H=(-i \sqrt{\alpha} \nabla+\gamma v)^{2}$ $+V(x)$. Obviously, the addition of a constant vector potential does not change the eigenvalues, and second order perturbation (i.e. first order in $\gamma^{2} v^{2}$ and second order in $-2 i \sqrt{\alpha} \gamma v \nabla$ ) yields (2.5) when choosing $v$ to be the canonical unit vectors $e_{a}$ and then summing over all $a=1, \ldots, d$. The author thanks R. Seiringer for indicating this proof.

Choosing $f$ appropriately in Theorem 2.1 we may now prove our main results.

Proof of Theorem 1.1. We note that for any $\alpha>0$ the operator $H(\alpha)$ has at most a finite number of negative eigenvalues. Obviously, the r.h.s. in 2.6 is negative. Making the dependence on the parameter $\alpha$ explicit we have therefore for all $z \leq 0$ the inequality

$$
\alpha \sum_{E_{j}(\alpha)<0}\left(z-E_{j}(\alpha)\right)^{2}-\frac{4}{d} \alpha^{2} \sum_{E_{j}(\alpha)<0}\left(z-E_{j}(\alpha)\right) T_{j}(\alpha) \leq 0 .
$$

The functions $E_{j}(\alpha)$ are non-positive, continuous and increasing. Furthermore, let $\infty \geq$ $\alpha_{1} \geq \alpha_{2} \geq \cdots>0$ denote the values at which $E_{j}(\alpha)$ appears. $E_{j}(\alpha)$ is continuously 
differentiable for $\alpha \neq \alpha_{k}$ and by the Feynman-Hellmann theorem

$$
\frac{d}{d \alpha} E_{j}(\alpha)=T_{j}(\alpha)
$$

Taking $z=0$, inequality (2.7) then reads

$$
\alpha \sum_{E_{j}(\alpha)<0}\left(-E_{j}(\alpha)\right)^{2}+\frac{2}{d} \alpha^{2} \frac{d}{d \alpha} \sum_{E_{j}(\alpha)<0}\left(-E_{j}(\alpha)\right)^{2} \leq 0 .
$$

For any $\alpha \in] \alpha_{N+1}, \alpha_{N}[$ the number of eigenvalues is constant and therefore

$$
\frac{d}{d \alpha}\left(\alpha^{d / 2} \sum_{E_{j}(\alpha)<0}\left(-E_{j}(\alpha)\right)^{2}\right) \leq 0
$$

proving the theorem.

Remark 2.3. Strictly speaking, the Feynman-Hellmann theorem only holds for nondegenerate eigenvalues. In the case of degenerate eigenvalues one has to take the right basis in the corresponding eigenspace and to change the numbering if necessary (see e.g. [14]).

Proof of Theorem 1.2 Choose $f(E)=e^{-t E}$ and $t>0$. Since $f^{\prime}(E)=-t f(E)$ is concave it follows that

$$
f^{\prime}\left(s E_{j}+(1-s) E_{k}\right) \geq s f^{\prime}\left(E_{j}\right)+(1-s) f^{\prime}\left(E_{k}\right) .
$$

Using the symmetry of $T_{j k}$ we get

$$
d \sum_{E_{j}(\alpha)} f\left(E_{j}(\alpha)\right)+2 \alpha \sum_{E_{j}(\alpha)} f^{\prime}\left(E_{j}(\alpha)\right) T_{j}(\alpha) \leq 0
$$

and we conclude as in the proof of Theorem 1.1

\section{Extensions and discussion}

It has already been shown in [6, 5, 7] that one can obtain trace inequalities for the functions $f(E)=(z-E)^{\sigma}$ with $\sigma \geq 2$. In fact we have the following result:

Corollary 3.1. Let $f: \mathbb{R} \rightarrow \mathbb{R}$ be a $C^{1}$ function with support on the negative half axis such that $f^{\prime}$ is concave. Under the conditions of Theorem 1.1 the mapping

$$
\alpha \mapsto \alpha^{d / 2} \sum_{E_{j}(\alpha)<0} f\left(E_{j}(\alpha)\right)
$$

is non-increasing for $\alpha>0$. In particular, for all $\sigma \geq 2$,

$$
\alpha \mapsto \alpha^{d / 2} \sum_{E_{j}(\alpha)<0}\left(-E_{j}(\alpha)\right)^{\sigma}
$$

is non-increasing for $\alpha>0$. Consequently,

$$
\alpha^{d / 2} \sum_{E_{j}(\alpha)<0}\left(-E_{j}(\alpha)\right)^{\sigma} \leq L_{\sigma, d}^{\mathrm{cl}} \int_{\mathbb{R}^{d}} V_{-}(x)^{\sigma+d / 2} d x .
$$


Proof. Since $f$ is of class $C^{1}$ we may rewrite the trace formula 2.1 as follows (note that $\left.T_{j j}=0\right)$ :

$$
d \sum_{E_{j}} f\left(E_{j}\right)+2 \alpha \sum_{E_{j}} \sum_{E_{k}} T_{j k} \int_{0}^{1} f^{\prime}\left(s E_{j}+(1-s) E_{k}\right) d s=0 .
$$

The concavity of $f^{\prime}$ implies that

$$
\int_{0}^{1} f^{\prime}\left(s E_{j}+(1-s) E_{k}\right) d s \geq \frac{1}{2} f^{\prime}\left(E_{j}\right)+\frac{1}{2} f^{\prime}\left(E_{k}\right) .
$$

Using the symmetry of $T_{j k}$ and $f^{\prime}(E)=0$ for $E \geq 0$ we get

$$
d \sum_{E_{j}(\alpha)<0} f\left(E_{j}(\alpha)\right)+2 \alpha \sum_{E_{j}(\alpha)<0} f^{\prime}\left(E_{j}(\alpha)\right) T_{j}(\alpha) \leq 0 .
$$

As in the proof of Theorem 1.1 we use the Feynman-Hellmann theorem to prove (3.1).

Remark 3.2. The sharp Lieb-Thirring inequality (3.3) follows also from (1.7) of Theorem 1.1 via the Aizenman-Lieb monotonicity principle [1]. However, the monotonicity of the mapping (3.2) is a stronger and new result.

Remark 3.3. Theorems 1.1 and 1.2 are also valid in the presence of magnetic fields, i.e. $H(\alpha)=-\alpha(-i \nabla+A(x))^{2}+V(x)$, and in the case of matrix valued potentials, since all commutation relations remain unchanged and the trace formula [2.1] still holds [6, 7].

Remark 3.4. We cannot expect that the monotonicity holds for moments with $\sigma<2$. For example, consider the $d$-dimensional harmonic oscillator with eigenvalues $E_{j}(\alpha)=$ $\sqrt{\alpha}\left(2 j_{1}+\cdots+2 j_{d}+d\right)$ for natural numbers $j_{1}, \ldots, j_{d}$. We want to study the behaviour of the eigenvalue moments

$$
S_{\sigma}(\alpha)=\sum_{E_{j}(\alpha)<1}\left(1-E_{j}(\alpha)\right)^{\sigma} .
$$

Then for all $\alpha \in\left[(d+2)^{-2}, d^{-2}\right]$ we have

$$
\alpha^{d / 2} S_{\sigma}(\alpha)=\alpha^{d / 2}(1-d \sqrt{\alpha})^{\sigma} .
$$

It is easy to see that the derivative of the above expression (with respect to $\alpha$ ) is strictly positive at $\alpha=(d+2)^{-2}$ for all $0 \leq \sigma<2$. This behaviour persists also for the sum of the first two eigenvalues. Indeed, for all $\alpha \in\left[(d+4)^{-2},(d+2)^{-2}\right]$ we have (taking into account the multiplicity of the second eigenvalue)

$$
S_{\sigma}(\alpha)=(1-d \sqrt{\alpha})^{\sigma}+d(1-(d+2) \sqrt{\alpha})^{\sigma} .
$$

Then the function $p_{\sigma}(\alpha):=\alpha^{d / 2} S_{\sigma}(\alpha)$ has a strictly positive derivative at $\alpha=(d+4)^{-2}$ for all $0 \leq \sigma<2$. Obviously, for $\sigma=2$ the derivatives at these points vanish.

Acknowledgments. The author would like to thank Evans M. Harrell II for fruitful discussions and many helpful comments. Valuable suggestions by R. Frank and E. H. Lieb are also gratefully acknowledged. 


\section{References}

[1] Aizenman, M., Lieb, E. H.: On semi-classical bounds for eigenvalues of Schrödinger operators. Phys. Lett. 66A, 427-429 (1978) MR 0598768

[2] Benguria, R., Loss, M.: A simple proof of a theorem by Laptev and Weidl. Math. Res. Lett. 7, 195-2003 (2000) Zbl 0963.34077 MR 1764316

[3] Blanchard, Ph., Stubbe, J.: Bound states for Schrödinger Hamiltonians: phase space methods and applications. Rev. Math. Phys. 35, 503-547 (1996) Zbl 0859.35101 MR 1405763

[4] de la Bretèche, R.: Preuve de la conjecture de Lieb-Thirring dans le cas des potentiels quadratiques strictement convexes. Ann. Inst. H. Poincaré Phys. Théor. 70, 369-380 (1999) Zbl 0938.35148 MR 1686426

[5] Harrell II, E. M., Hermi, L.: Differential inequalities for Riesz means and Weyl-type bounds for eigenvalues. J. Funct. Anal. 254, 3173-3191 (2008) Zbl 1147.35062 MR 2418623

[6] Harrell II, E. M., Stubbe, J.: On trace identities and universal eigenvalue estimates for some partial differential operators. Trans. Amer. Math. Soc. 349, 1797-1809 (1997) Zbl 0887.35111 MR 1401772

[7] Harrell II, E. M., Stubbe, J.: Universal bounds and semiclassical estimates for eigenvalues of abstract Schrödinger operators. SIAM J. Math. Anal., to appear

[8] Helffer, B., Robert, D.: Riesz means of bound states and semi-classical limit connected with a Lieb-Thirring conjecture I, II. Asymptotic Anal. 3, 91-103 (1990), Ann. Inst. H. Poincaré Phys. Théor. 53, 139-147 (1990) Zbl 0717.35062 Zbl 0728.35078 MR 1061661 MR 1079775

[9] Hundertmark, D.: Some bound state problems in quantum mechanics. In: Spectral Theory and Mathematical Physics: A Festschrift in Honor of Barry Simon's 60th Birthday, Proc. Sympos. Pure Math. 76, part 1, F. Gesztesy et al. (eds.), Amer. Math. Soc., 463-496 (2007) Zbl 1126.81025 MR 2310215

[10] Laptev, A., Weidl, T.: Sharp Lieb-Thirring inequalities in high dimensions. Acta Math. 184, 87-111 (2000) Zbl 1142.35531 MR 1756570

[11] Laptev, A., Weidl, T.: Recent results on Lieb-Thirring inequalities. In: Journées "Équations aux Dérivées Partielles" (La Chapelle sur Erdre, 2000), exp. XX, Univ. Nantes, Nantes, 14 pp. (2000) Zbl 1135.81337 MR 1775696

[12] Lieb, E. H., Thirring, W.: Inequalities for the moments of the eigenvalues of the Schrödinger Hamiltonian and their relation to Sobolev inequalities. In: Studies in Mathematical Physics, Essays in Honor of Valentine Bargmann, Princeton, 269-303 (1976) Zbl 0342.35044

[13] Simon, B.: Trace Ideals and Their Applications. 2nd ed., Math. Surveys Monogr. 120, Amer. Math. Soc. (2005) Zbl 1074.47001 MR 2154153

[14] Thirring, W.: Quantum Mathematical Physics: Atoms, Molecules and Large Systems. 2nd ed., Springer (2002, revised 2003), 149-150 MR 2133871 